Table 2.

\begin{tabular}{|c|c|c|c|c|c|c|}
\hline Parameter & G 1 & G 2 & C & $\begin{array}{c}p \\
\text { G1 vs G2 }\end{array}$ & $\begin{array}{c}p \\
\text { G1 vs } C\end{array}$ & $\begin{array}{c}\mathrm{p} \\
\mathrm{G} 2 \text { vs } \mathrm{C}\end{array}$ \\
\hline Pregnancies $\wedge \Uparrow$ & 29 & 70 & 88 & $<0.0001$ & $<0.05$ & $<0.05$ \\
\hline Children/mother^ & 1.31 & 2.59 & 2.14 & - & - & - \\
\hline Age at conception $y^{\pi}$ & $34 ; 6$ & $23 ; 4.5$ & $23 ; 6.5$ & $<0.0001$ & $<0.0001$ & $<0.05$ \\
\hline $\mathrm{m} ; \mathrm{IQR}(\min -\mathrm{max})$ & $(28-44)$ & $(17-38)$ & $(16-39)$ & & & \\
\hline TTP $>12$ months $(\%)^{\varepsilon}$ & 37.93 & 48.57 & 30.68 & $<0.0001$ & $<0.05$ & $>0.05$ \\
\hline Births $\wedge$ ^ & 28 & 53 & 67 & $<0.0001$ & $<0.0001$ & $>0.05$ \\
\hline Spontaneous abortions $\wedge^{\wedge}$ & 10 & 14 & 22 & $<0.0001$ & $<0.0001$ & $>0.05$ \\
\hline Stillbirths^ & 0 & 1 & 0 & - & - & - \\
\hline 'C 'section delivery $(\%)$ & 21.42 & 1.85 & 1.51 & $<0.0001$ & $<0.0001$ & $>0.05$ \\
\hline Premature delivery ${ }^{\varepsilon}(\%)$ & 7.14 & 5.66 & 3.07 & $>0.05$ & $>0.05$ & $>0.05$ \\
\hline Eclampsia (\%) & 3.57 & 0 & 0 & - & - & - \\
\hline Birth weight $g(\text { mean } \pm S D)^{\natural}$ & $2950 \pm 461$ & $3250 \pm 652$ & $3500 \pm 463$ & $<0.05$ & $<0.0001$ & $<0.05$ \\
\hline Birth weight < 2500g (\%) & 7.14 & 13.33 & 1.49 & - & - & - \\
\hline Malformations ${ }^{\wedge}$ & 0 & 0 & 1 & - & - & - \\
\hline Transfer to NICU^ & 0 & 1 & 1 & - & - & - \\
\hline Other child pathologies^^ & 2 (autism) & 0 & 0 & - & - & - \\
\hline
\end{tabular}

^- number, $y$ - years, g-gram, NICU- newborn intensive care unit, " Mann Whitney U test,

${ }^{\varepsilon}$ Fisher's exact test, $\mathrm{m}$-median

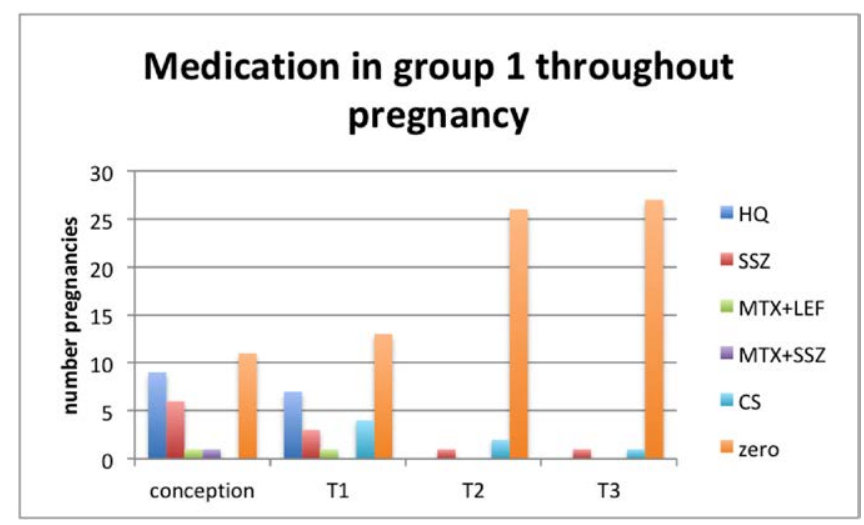

Fig 1:

Conclusion: Current pregnancy management guidelines implementations in RA assure a good pregnancy course in patients that have achieved remission and LDA status. RA patients show lower fertility and more miscarriages in comparison to both preclinical RA and general population. Newborns from RA mothers are healthy and show a normal gestational age/weight at delivery, yet smaller family sizes were observed. Preclinical RA does not influence fertility, pregnancy outcome and family size. Newborns belonging to G1 and G2 are smaller in comparison to general population but true hypotrophy is a rare event.

References:

[1] Rom AL, Wu CS, Olsen J, et al. Fetal growth and preterm birth in children exposed to maternal or paternal rheumatoid arthritis: a nationwide cohort study. Arthrtis Rheum 2014; 66(12):3265-73.

[2] de Man YA, Hazes JM, van der Heide $H$, Association of higher rheumatoid arthritis disease activity during pregnancy with lower birth weight: results of a national prospective study. Arthritis Rheum 2009;60:3196-206.

Disclosure of Interests: None declared

DOI: 10.1136/annrheumdis-2020-eular.2545

\section{FRI0071 ANALYSIS OF DATA GAPS IN RHEUMATOID ARTHRITIS}

G. Candelas ${ }^{1}$, L. Silva-Fernández ${ }^{2}$, M. Montoro ${ }^{3}$, A. Hernández ${ }^{4}$, J.

R. Maneiro ${ }^{5}$, V. Villaverde ${ }^{6}$, L. Carmona ${ }^{7}$, E. Loza ${ }^{7}$, S. Gómez ${ }^{3}$, M. Valderrama ${ }^{3}$ A. Ortiz ${ }^{8} .{ }^{1}$ Hospital Clínico San Carlos, Rheumatology, Madrid, Spain;

${ }^{2}$ Complexo Hospitalario Universitario de A Coruña, Servicio de Reumatología, A Coruña, Spain; ${ }^{3}$ Pfizer, Medical Department, Madrid, Spain; ${ }^{4}$ Hospital Virgen del Puerto, Rheumatology, Plasencia, Spain; ${ }^{5}$ Complejo Hospitalario Universitario de Pontevedra, Pontevedra, Spain; ${ }^{6}$ Hospital Universitario de Móstoles, Madrid, España, Rheumatology, Móstoles, Spain; ${ }^{7}$ Instituto de salud Musculoesquelética, Madrid, Spain; ${ }^{8}$ Hospital Universitario de la Princesa, Instituto de Investigación Sanitaria la Princesa (IIS-IP), Madrid, Spain

Background: Although ideally Recommendations for the management of rheumatoid arthritis (RA) should be supported by the highest level of evidence, many of which are based on "expert opinion." This means that there are knowledge gaps to which a part of the research efforts in this disease should be directed.

\section{Objectives:}

1.- Analyze the causes of the low level of evidence in some of the recommendations on diagnosis and management of RA in the main published documents 2.- Identify the knowledge gaps that justify said low level of evidence

3.- Design actions to respond to the knowledge gaps identified.

Methods: Qualitative study. A group of six experts in systematic review of the literature was selected. Fourteen documents of national and international recommendations on RA (EULAR, ACR and SER) of the last 5 years were analyzed by a peer review. They selected recommendations with low level of evidence (Oxford 4 and 5) / grade of recommendation (C and D), and classified by areas (diagnosis, monitoring, treatment, others) and then possible causes of low level of evidence were analyzed. These were submitted to a Delphi to select the $10 \mathrm{rec}-$ ommendations in which participants considered it more critical to obtain quality evidence. Subsequently, actions were proposed to improve the levels of evidence in general and, through the PICOS structure (population, intervention, comparator, study design) specific studies were proposed to respond to the issues raised in these 10 recommendations

Results: 185 recommendations were found that had a low level of evidence / grade of recommendation, most related to the treatment of RA. The two most frequent causes of this low level of evidence and / or the degree of recommendation were the absence of studies and an incorrect classification of the level of evidence and / or degree of recommendation. In addition, other reasons and methodological barriers were found for which nine critical recommendations were finally selected for which new PICOs were developed with which to propose targeted research projects

Conclusion: It is necessary to improve the methodological approach in the RA recommendations guidelines to correct errors and fill gaps with appropriate studies.

Table 1. Actions to increase the level of evidence / recommendation.

\section{\# Action}

1 Prioritization of research towards knowledge gaps with the design and development of specific studies

2 Increase knowledge of experts in the methodology of consensus documents (including RSL, formulation of recommendations, etc.)

Supervision of the entire process by expert methodologists, to ensure a correct allocation of the levels of evidence and degree of recommendation

4 Review and select those topics that are really of interest and should be reviewed and can be answered

5 Expert opinion should never become a recommendation, but will be included in the text that accompanies that recommendation.

$6 \quad$ Clear syntax will be used and short recommendations will be made

7 Establishment and application of homogeneous criteria to formulate recommendations

Key words: Rheumatoid arthritis, recommendations, data gaps

Disclosure of Interests: gloria candelas: None declared, Lucía Silva-Fernández: None declared, Maria Montoro Employee of: Pfizer employee, Abad Hernández: None declared, Jose Ramón Maneiro: None declared, Virginia Villaverde: None declared, Loreto Carmona Grant/research support from: Novartis Farmaceutica, SA, Pfizer, S.L.U., Merck Sharp \& Dohme España S.A., Roche Farma, S.A, Sanofi Aventis, AbbVie Spain, S.L.U., and Laboratorios Gebro Pharma, SA (All trhough institution), Estíbaliz Loza Grant/research support from: Roche, Pfizer, Abbvie, MSD, Novartis, Gebro, Adacap, Astellas, BMS, Lylly, Sanofi, Eisai, Leo, Sobi, Susana Gómez Employee of: Pfizer employee, Monica Valderrama Consultant of: Pfizer employee, Ana Ortiz: None declared

DOI: 10.1136/annrheumdis-2020-eular.6574

\section{FRI0072 DISCONTINUATION OF DMARD USE IN RHEUMATOID ARTHRITIS PATIENTS WITH LUNG DISEASE}

S. Pedro ${ }^{1}$, T. Mikuls ${ }^{2,3}$, J. Zhuo ${ }^{4}$, K. Michaud ${ }^{1,2} .{ }^{1}$ FORWARD, The National Databank for Rheumatic Diseases, Wichita, KS, United States of America; ${ }^{2}$ University of Nebraska Medical Center, Omaha, NE, United States of America; ${ }^{3}$ VA Nebraska-Western lowa Health Care System, Omaha, NE, United States of America; ${ }^{4}$ Bristol-Myers Squibb, Princeton, NJ, United States of America

Background: Pulmonary manifestations such as interstitial lung disease (ILD) and chronic obstructive pulmonary disease (COPD) are frequent extra-articular features that carry a poor prognosis in Rheumatoid Arthritis (RA). Little data is available on how RA patients (pts) with pulmonary disease are managed in realworld settings. 
Objectives: To assess treatment patterns and DMARD discontinuation in RA patients with comorbid lung disease in comparison with other RA patients. Methods: The study included RA Patients enrolled in the Forward Databank with $\geq 1$ year observation after 2000 initiating a DMARD. Forward is a large longitudinal rheumatic disease registry in the US. RA patients' diagnoses were rheumatologist-confirmed, and every 6 months participants completed comprehensive questionnaires regarding symptoms, disease outcomes, medications, and clinical events. Lung disease (LD+) was defined as at least one of the following: emphysema, asthma, bronchitis, COPD, pleural effusion, fibrosis of the lung, "RA lung", or ILD, the later classified by ICD9 codes (England 2019). DMARDs were categorized hierarchically into four groups: csDMARDs, TNFi and NTNFi (bDMARDs), and tsDMARDs. Percentage of patients who initiated different DMARDs were reported for pts with LD+/LD-. Discontinuation was analyzed by Kaplan Meier (KM) curves, log-ranks tests, and Cox regression models using time-varying covariates. Best models were created using backward selection models ( $10 \%$ probability of removal) and pre-defined clinical models.

Results: Of the 21,525 eligible RA patients, $13.8 \%$ had LD+ at the time they initiated a DMARD (follow-up: 69,597 pt-yrs (median $1.9 \mathrm{yrs} / \mathrm{pt}$ )). LD+ patients tended to have more severe RA outcomes and comorbidities. MTX-monotherapy $(48 \%$ vs $44 \%, p<0.001)$ and NTNFi were initiated more frequently in LD+ pts with lower use of TNFi (Figure). DMARD discontinuation rates were higher among LD+ patients for all DMARD groups, but KM curves were only significantly different for csDMARDs and TNFi. Different HRs for LD+ were found depending on the model used ranging from 1.18 to 1.28 , and all models revealed an increased risk of discontinuation for LD+ patients. Compared to csDMARDs, TNFi were more often discontinued (Table). Other variables associated with an increased risk of discontinuation included: $\mathrm{HAQ}$, Rheumatoid Disease (RD) comorbidity index, pain, prior bDMARDs, and csDMARDs.

Conclusion: Different DMARD treatment patterns were found for LD+ patients, who tended to initiate more csDMARD and NTNFi and less likely to initiate a TNFi. LD+ patients were at a higher risk of discontinuation irrespectively of the DMARD treatment, but with greater risk for TNF users.

References:

[1] England BR, et al. Arth Care Res. doi:10.1002/acr.24043.

Figure. DMARD treatment initiators by disease group

Table. Cox models for DMARD discontinuation by stepwise (removal probability $10 \%$ ) and clinical models including DMARD treatment.

\begin{tabular}{lccc}
\hline $\begin{array}{l}\text { Model of DMARD } \\
\text { persistence }\end{array}$ & $\begin{array}{c}\text { Model 1- Stepwise- } \\
\text { Without drugs }\end{array}$ & Model 2 & Model 3 - \\
LD+ vs LD- & 1.18 & 1.28 & Clinical \\
& $(1.08-1.29)$ & $(1.13-1.45)$ & 1.20 \\
TNF vs csDmard & & 1.32 & $1.08-1.34)$ \\
& & $(1.08-1.63)$ & $(1.04-1.44)$ \\
NTNF vs csDmard & 1.13 & 1.13 \\
& & $(0.83-1.52)$ & $(0.90-1.41)$ \\
tsDmard vs csDmard & 1.30 & 1.02 \\
& & $(0.65-2.60)$ & $(0.64-1.62)$
\end{tabular}

*Best models searched/Clinical adjusted for LD+/LD-, DMARDs, age, sex, education, HAQ disability, RD comorbidity index, smoking, pain, glucocorticoids, year of entry, prior bDMARDs and csDMARDs counts and MRC breath scale.

Disclosure of Interests: Sofia Pedro: None declared, Ted Mikuls Grant/ research support from: Horizon Therapeutics, BMS, Consultant of: Pfizer, Joe Zhuo Shareholder of: Bristol-Myers Squibb, Employee of: Bristol-Myers Squibb, Kaleb Michaud: None declared

DOI: 10.1136/annrheumdis-2020-eular.5452

\section{FRI0073 SEROLOGICAL PREDICTORS OF THE SEVERITY OF RHEUMATOID ARTHRITIS RELATED INTERSTITIAL LUNG DISEASE}

S. Rajalingham ${ }^{1}$, S. S. Shaharir ${ }^{1}$, R. Sridharan ${ }^{1} .{ }^{1}$ Universiti Kebangsaan Malaysia Medical Centre, Kuala Lumpur, Malaysia

Background: The most common extra-articular manifestation of rheumatoid arthritis(RA) is interstitial lung disease (ILD). Although pulmonary manifestations in RA encompass the main airway, parenchyma, vasculature and pleura, ILD in particular, is associated with reduced survival. Up to $10 \%$ of RA patients suffer from clinically significant ILD while a substantial proportion have abnormal CT chest findings despite being asymptomatic. There are various biochemical and serological markers to predict the severity of the joints in RA. However, the clinical and laboratory determinants of RA related ILD (RA-ILD) are not well defined owing to the paucity of research data in this regard.
Objectives: The main objective of this study is to determine the correlation between the rheumatoid factor (RF) serotypes and the severity of RA-ILD based on computer tomography (CT) findings.

Methods: We recruited a total of 100 RA patients who were tested for IgA RF, IgG RF and IgM RF and had high resolution CT chest performed. Participants were aged above 18 years, met the 2010 ACR/EULAR RA criteria, had RA for more than 6 months, were non-smokers, not pregnant and had no known chronic lung disease or lung malignancy based on their medical records. Seventy-two patients had ILD changes on HRCT of the chest and were included in this study. The CT images were scored based on a scoring system proposed by Kazerooni et al. Ground glass opacities represented the alveolar findings whereas honeycombing and septal thickening were the interstitial findings. The ground glass and fibrosis scores were on a scale of $0-5$, with higher scores for greater involvement of the lobes. For each subject, the maximum ground glass and fibrosis scores were 25 , respectively.

Results: The frequency of RF positivity was comparable across the 3 serotypes (83.33-84.72\%). The correlation between the clinical variables and the CT scores are listed in Table 1.We found that the the CT scores for ground glass showed significant positive correlation with disease duration $(p=0.047)$ and $\lg A$ RF levels $(p<0.050)$ whereas the fibrosis scores had significant relationship with multiple clinical covariates i.e age $(p=0.004)$, disease duration $(p=0.042)$, IgA RF levels $(p<0.050)$, IgG RF levels $(p=0.041)$ and anti-CCP levels $(p=0.006)$. On multivariate analysis, only IgA RF levels remained significantly $(p<0.05$, standardized beta coefficient $=0.604$ ) associated with the ground glass scores. As for the fibrosis scores, IgA RF levels and age were independent predictors based on multivariate analysis after adjusting for confounders, with $p$ scores of $<0.05$ and 0.02 , respectively.

Table 1. Correlation of CT scores with clinical covariates and antibodies

\begin{tabular}{|c|c|c|c|c|}
\hline \multirow[t]{2}{*}{ Parameters } & \multicolumn{2}{|c|}{ Ground glass } & \multicolumn{2}{|c|}{ Fibrosis } \\
\hline & $r$ & $p$ value & $r$ & $p$ value \\
\hline Age & 0.229 & 0.053 & 0.334 & 0.004 \\
\hline Disease duration & 0.235 & 0.047 & 0.240 & 0.042 \\
\hline Total MSS & 0.058 & 0.628 & 0.224 & 0.145 \\
\hline Cumulative Methotrexate dose & 0.039 & 0.748 & 0.098 & 0.413 \\
\hline $\lg A$ & 0.608 & $<0.05$ & 0.576 & $<0.05$ \\
\hline $\lg M$ & 0.053 & 0.660 & 0.019 & 0.873 \\
\hline $\lg G$ & 0.183 & 0.124 & 0.241 & 0.041 \\
\hline Anti CCP & 0.117 & 0.328 & 0.319 & 0.006 \\
\hline
\end{tabular}

Conclusion: The IgA RF was the only serotype which was independently associated with the severity of RA-ILD.

References:

[1] Bongartz, T., Nannini, C., Medina-Velasquez, Y. F., Achenbach, S. J., Crowson, C. S., Ryu,et al. (2010). Incidence and mortality of interstitial lung disease in rheumatoid arthritis: a population-based study. Arthritis and rheumatism, 62(6), 1583-1591.

[2] Dawson, J. K., Fewins, H. E., Desmond, J., Lynch, M. P., \& Graham, D. R. (2001). Fibrosing alveolitis in patients with rheumatoid arthritis as assessed by high resolution computed tomography, chest radiography, and pulmonary function tests. Thorax, 56(8), 622-627.

[3] Kazerooni, E. A., Martinez, F. J., Flint, A., Jamadar, D. A., Gross, B. H.,et al (1997). Thin-section CT obtained at 10-mm increments versus limited threelevel thin-section CT for idiopathic pulmonary fibrosis: correlation with pathologic scoring. AJR Am J Roentgenol, 169(4), 977-983.

[4] van der Helm-van Mil, A. H., \& Huizinga, T. W. (2012). The 2010 ACR/ EULAR criteria for rheumatoid arthritis: do they affect the classification or diagnosis of rheumatoid arthritis? Annals of the rheumatic diseases, 71(10), 1596-1598

Disclosure of Interests: Sakthiswary Rajalingham Speakers bureau: Pfizer (500USD), Syahrul Sazliyana Shaharir: None declared, Radhika Sridharan: None declared

DOI: 10.1136/annrheumdis-2020-eular.218

\section{FRI0074 UNDERLYING PROBLEMS AND IMPACT OF DIFFICULT TO-TREAT RHEUMATOID ARTHRITIS: PRELIMINARY RESULTS OF A CROSS-SECTIONAL CASE-CONTROL STUDY}

N. M. T. Roodenrijs ${ }^{1}$, M. C. Van der Goes ${ }^{1,2}$, P. Welsing ${ }^{1}$, J. Tekstra $^{1}$, F. Lafeber ${ }^{1}$ J. W. G. Jacobs ${ }^{1}$, J. M. Van Laar ${ }^{1} .{ }^{1}$ University Medical Center, Department of Rheumatology \& Clinical Immunology, Utrecht, Netherlands; ${ }^{2}$ Meander Medical Center, Department of Rheumatology, Amersfoort, Netherlands 